\title{
Azimuthal asymmetry in the risetime of the Surface Detector signals of the Pierre Auger Observatory
}

\author{
I.A. Minaya ${ }^{* a}$ for the Pierre Auger Collaboration ${ }^{b}$ \\ ${ }^{a}$ Department of Atomic, Molecular and Nuclear Physics, Complutense University of Madrid, \\ Madrid, Spain \\ ${ }^{b}$ Observatorio Pierre Auger, Av. San Martín Norte 304, 5613 Malargüe, Argentina \\ E-mail: auger_spokespersons@fnal.gov \\ Full author list: http://Www.auger.org/archive/authors_2015_06.htm
}

\begin{abstract}
The azimuthal asymmetry in the risetime of signals in Auger surface detector stations is a source of information on shower development. The azimuthal asymmetry is due to a combination of the longitudinal evolution of the shower and geometrical effects related to the angles of incidence of the particles into the detectors. The magnitude of the effect depends upon the zenith angle and state of development of the shower and thus provides a novel observable sensitive to the mass composition of cosmic rays above $3 \times 10^{18} \mathrm{eV}$. By comparing measurements with predictions from shower simulations, we find for both of our adopted models of hadronic physics (QGSJetII04 and Epos-LHC) an indication that the mean cosmic-ray mass increases with energy, as has been inferred from other studies. However the absolute values derived for the mass are dependent on the shower model and on the range of distance from the shower core selected. Thus the method has uncovered further deficiencies in our understanding of shower modelling that must be resolved before the mass composition can be inferred from $(\sec \theta)_{\max }$.
\end{abstract}

The 34th International Cosmic Ray Conference,

30 July- 6 August, 2015

The Hague, The Netherlands

\footnotetext{
* Speaker.
} 


\section{Introduction}

The measurement of the mass composition of ultra-high energy (UHE) cosmic rays ( $E \gtrsim 10^{18}$ $\mathrm{eV}$ ) is one of the greatest challenges in this field, not only due to the large fluctuations resulting from the stochastic nature of the extensive air-shower (EAS) development but also because it is necessary to make assumptions about the hadronic physics in regions of phase space not covered by measurements at accelerators. The Pierre Auger Observatory [四] uses two complementary techniques for the detection of EAS from UHE cosmic rays: a Fluorescence Detector (FD) that directly registers their longitudinal development and a Surface Detector Array (SD) that samples the shower tail by means of water-Cherenkov detectors. Although the FD provides a well-established technique for mass composition via the direct measurement of the depth of shower maximum, $X_{\max }$ [[D, (B]], its duty cicle is $<13 \%$. Therefore different techniques using the SD, which operates $\sim 100 \%$ of time, are being developed [四, [5, 目]. This paper is focused on an SD observable that is related to the azimuthal asymmetry found in the risetime of the signals with respect to the direction of the incoming air-shower. This asymmetry in its turn is related to the stage of the shower development and, as will be shown, has the potential to give alternative insights into the matching of data to mass and hadronic models.

\section{Concept of azimuthal asymmetry in the risetime}

The Auger water-Cherenkov detectors measure the spread in arrival times of the signals produced by the different components of an EAS. The risetime $t_{1 / 2}$, defined as the time of increase from $10 \%$ to $50 \%$ of the total integrated signal, is a useful parameter in composition studies. While in vertical showers the SD signals show a perfect circular symmetry around the polar angle $\zeta$ in the shower plane, in inclined showers, particles reaching late detectors $(\pi / 2<\zeta<-\pi / 2$, see Fig. W) traverse longer atmospheric paths than those arriving to early detectors $(-\pi / 2<\zeta<\pi / 2)$. Consequently for inclined showers, both the magnitude and risetime of the signals depend on $\zeta$. The method described here uses the above-mentioned azimuthal asymmetry.

The observed azimuthal asymmetry is due to two effects. On the one hand, a contribution comes from the quenching of the electromagnetic signal. Since the particles that reach late detectors traverse longer atmospheric paths, we expect a bigger attenuation of electrons and photons as compared to early detectors. On the other hand, there are also contributions to the asymmetry from geometrical effects. In this case, not only is the electromagnetic component important, but muons also play a role. Although track length and solid angle effects are compensated by the near-cubical design of the detectors, the angular distributions of muons impinging on the detectors are different, as late detectors record more muons emitted closer to the shower axis. Geometrical effects predominate at low zenith angles, while for showers with $\theta>30^{\circ}$ attenuation effects are the main contribution.

The azimuthal asymmetry of the $t_{1 / 2}$ depends on the distance to the core $r$ in the plane of the shower front, since the larger is $r$ the larger is the difference in atmospheric paths between early and late stations. In addition this asymmetry depends on the zenith angle of the shower, $\theta$. No asymmetry is expected for vertical showers: it grows with $\theta$ and finally this trend reaches a point where the EM component is quenched due to the longer atmospheric path travelled and the shower 


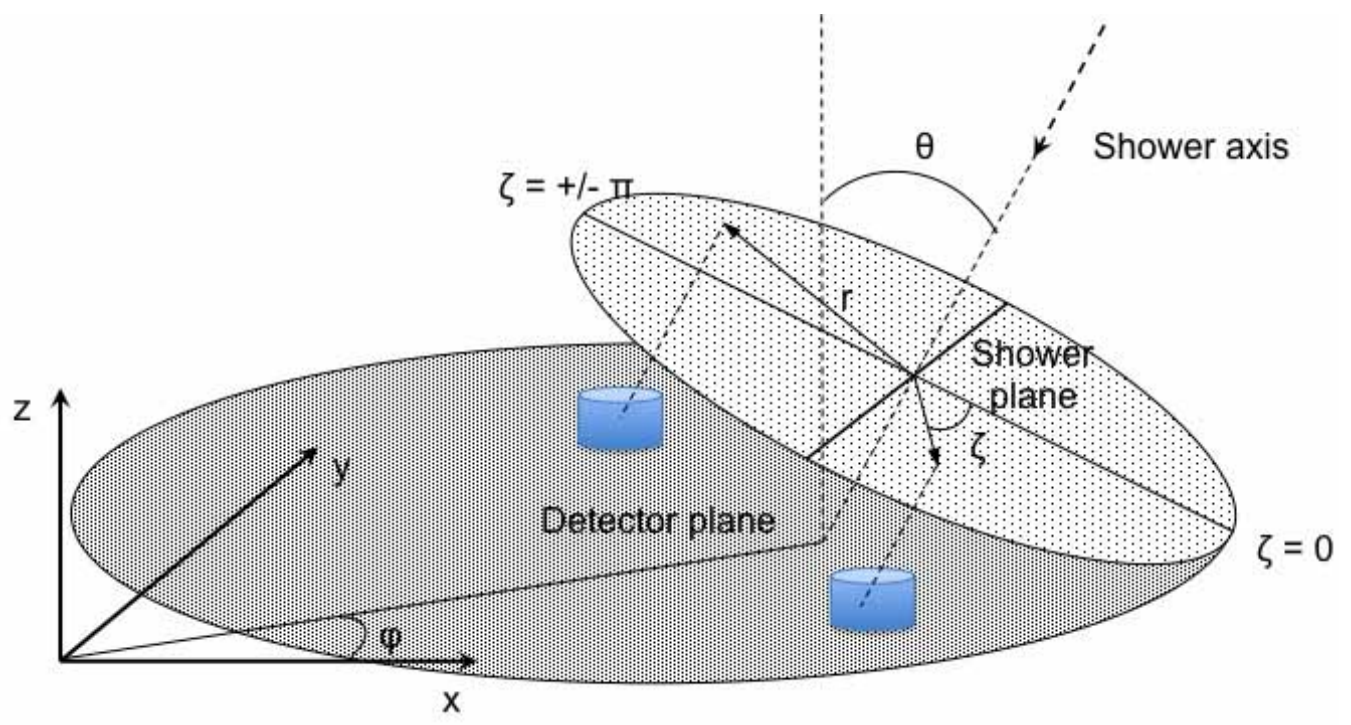

Figure 1: Schematic view of the shower geometry with the notation used in this paper. The incoming direction of the primary particle defines two regions: early $(-\pi / 2<\zeta<\pi / 2)$ and late $(\pi / 2<\zeta<-\pi / 2)$. Note the different amount of atmosphere traversed by the particles reaching the detectors in each region.

is mainly composed of penetrating muons. As will be demonstrated, for a given primary energy $E$, the time asymmetry dependence upon $\sec \theta$ shows a maximum which is sensitive to the average primary mass.

\section{Azimuthal asymmetry analysis using Auger data}

For the present analysis we have used data collected with the SD of the Pierre Auger Observatory from 01.01 .04 to 29.10 .14 with $E>3 \times 10^{18} \mathrm{eV}$ and $\theta<62^{\circ}$ to assure $100 \%$ detection efficiency, and with $\theta>30^{\circ}$ where the contribution of the attenuation to the azimuthal asymmetry dominates over other sources. These events are required to satisfy the standard trigger levels described in [प]. Additionally, quality cuts at station level have been applied: the recorded signal must be larger than 10 VEM (1 VEM is the signal produced by a vertical and central through-going muon) to assure $100 \%$ probability for single-detector triggering, and stations are required to be at a core distance $500<r<2000 \mathrm{~m}$ to assure an accurate determination of $t_{1 / 2}$. After application of these cuts a total of 191534 signals from 54584 events remain.

The first step in the analysis is to measure the azimuthal asymmetry of the $t_{1 / 2}$ for fixed $E$ and $\theta$ values. This measurement cannot be done on a shower-by-shower basis because it is not possible to sample the whole range of the polar angle, from early to late regions, in a single event. Thus, a statistical approach is applied to characterize the azimuthal asymmetry of $t_{1 / 2}$ using all the stations from the events at a given $E$ and $\theta$. To this end we use the variable $t_{1 / 2} / r$ since in this way the risetime information of all stations within given $E$ and $\theta$ bins can be used, independently of the core distance, to compute an average $\left\langle t_{1 / 2} / r\right\rangle$ value as a function of $\zeta$. Since the asymmetry depends on $r$, the analysis has been carried out independently for two $r$-intervals, i.e., $500-1000 \mathrm{~m}$ and 1000 - $2000 \mathrm{~m}$ each containing a similar number of signals and events. As an example, we show in the 

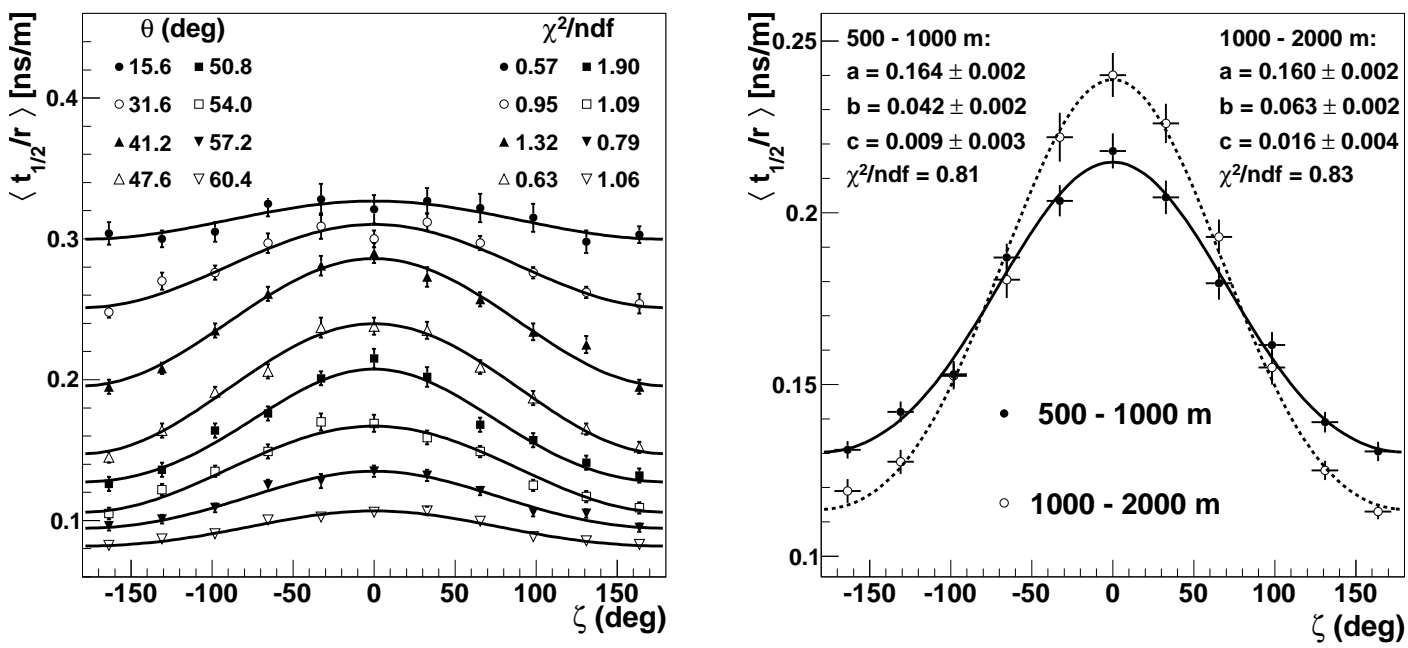

Figure 2: Asymmetry plots (dependence of $\left\langle t_{1 / 2} / r\right\rangle$ vs the polar angle in the shower plane $\zeta$ ) for different cases. Left panel: for $\log (E / e V)=19.2-19.5$ in the $500-1000 \mathrm{~m}$ interval at different $\theta$ bins. Right panel: for $\log (E / \mathrm{eV})=19.0-19.2$ in the two chosen core distance intervals with $\theta=51^{\circ}$; results of the fitted parameters (see text) are shown for each $r$-interval.

left panel of Fig. $\square\left\langle t_{1 / 2} / r\right\rangle$ vs $\zeta$ for $19.2<\log (E / e V)<19.5$ and eight $\theta$ values. For each $\theta$ band the data are fitted to the function $\left\langle t_{1 / 2} / r\right\rangle=a+b \cos \zeta+c \cos ^{2} \zeta$. The asymmetry with respect to $\zeta$ is evident and the ratio $b /(a+c)$, the so-called asymmetry factor, will be used to quantify the asymmetry. In right panel of Fig. $\square\left\langle t_{1 / 2} / r\right\rangle$ vs $\zeta$ is displayed for both core distance intervals for showers with $\log (E / \mathrm{eV})=19.0-19.2$ and $\theta=51^{\circ}$. As can be observed, the amplitude of the asymmetry for $500-1000 \mathrm{~m}$ is smaller. This is due to the fact that, close to the core, there is a higher electromagnetic contribution to the risetime and a smaller difference in the paths travelled by the particles.

Next, this asymmetry factor is represented as a function of atmospheric depth, measured by $\sec \theta$. For each energy interval, $b /(a+c)$ vs $\ln (\sec \theta)$ is fitted to a Gaussian function to obtain its maximum. An example is shown in Fig. [] for the same energy bin in each $r$-interval. The value of $\sec \theta$ for which the asymmetry parameter maximizes, $(\sec \theta)_{\max }$, will be used as the observable for mass composition. Once the value of $(\sec \theta)_{\max }$ for each energy bin has been obtained in each core distance interval, we can study the evolution of the dependence of $(\sec \theta)_{\max }$ on the primary energy. In Fig. 团 the result for both $r$-intervals is shown. As expected, for a given energy, $(\sec \theta)_{\max }$ is larger in the interval $500-1000 \mathrm{~m}$ than in the $1000-2000 \mathrm{~m}$ one since closer to the core the asymmetry is smaller, and thus, the zenith angle at which the muon component starts to dominate (and the asymmetry starts to decrease) is higher. Note that, in principle, this dependence on the radial interval should not limit the capability of the asymmetry method for mass analysis provided that Monte Carlo simulations follow the same behavior. This will be discussed in section $\theta$.

A first estimation of the systematic uncertainty of $(\sec \theta)_{\max }$ has been carried out for both core distance intervals. For that, we have taken into account the effect of the uncertainty in the reconstruction of the shower core, in the risetime and also the effect of the energy scale uncertainty, 
500 - 1000 m
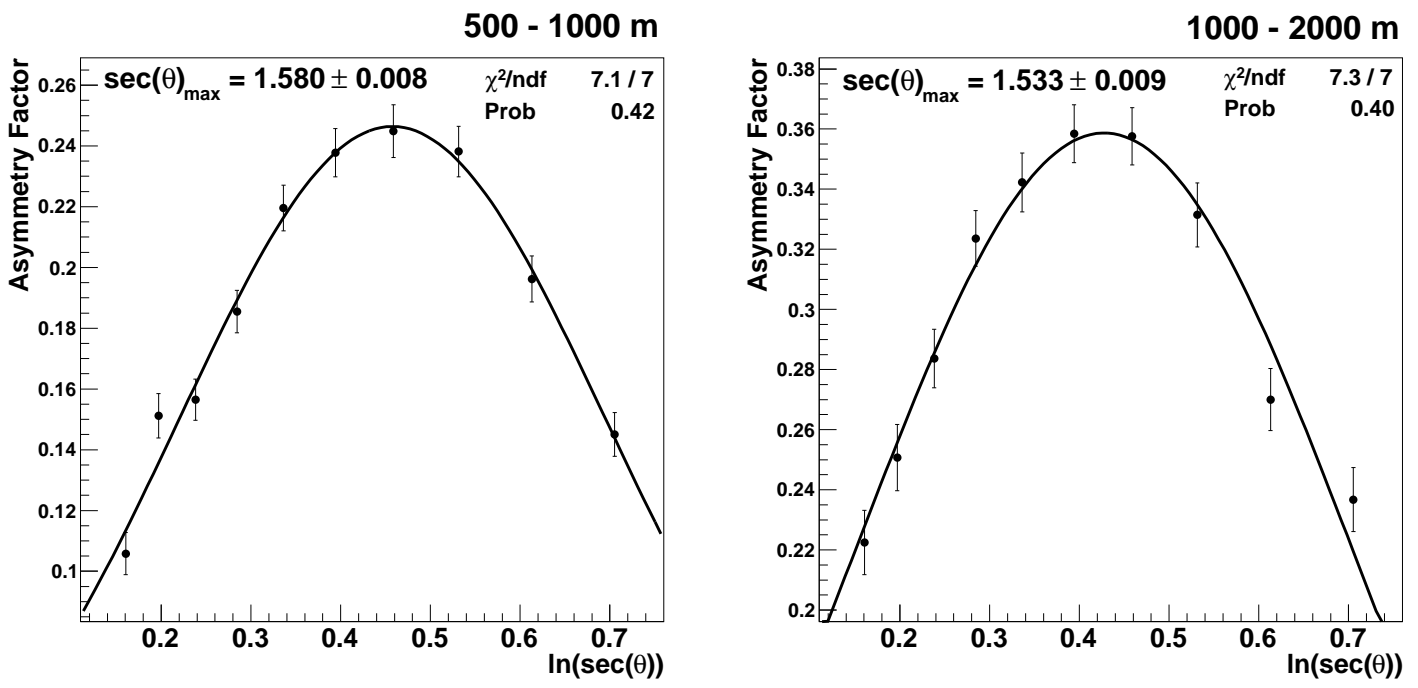

Figure 3: Longitudinal development of the asymmetry factor $(b /(a+c)$, see text) for $\log (E / e V)=19.0-19.2$ in both core distance intervals (500 - $1000 \mathrm{~m}$ in the left panel, $1000-2000 \mathrm{~m}$ in the right panel).

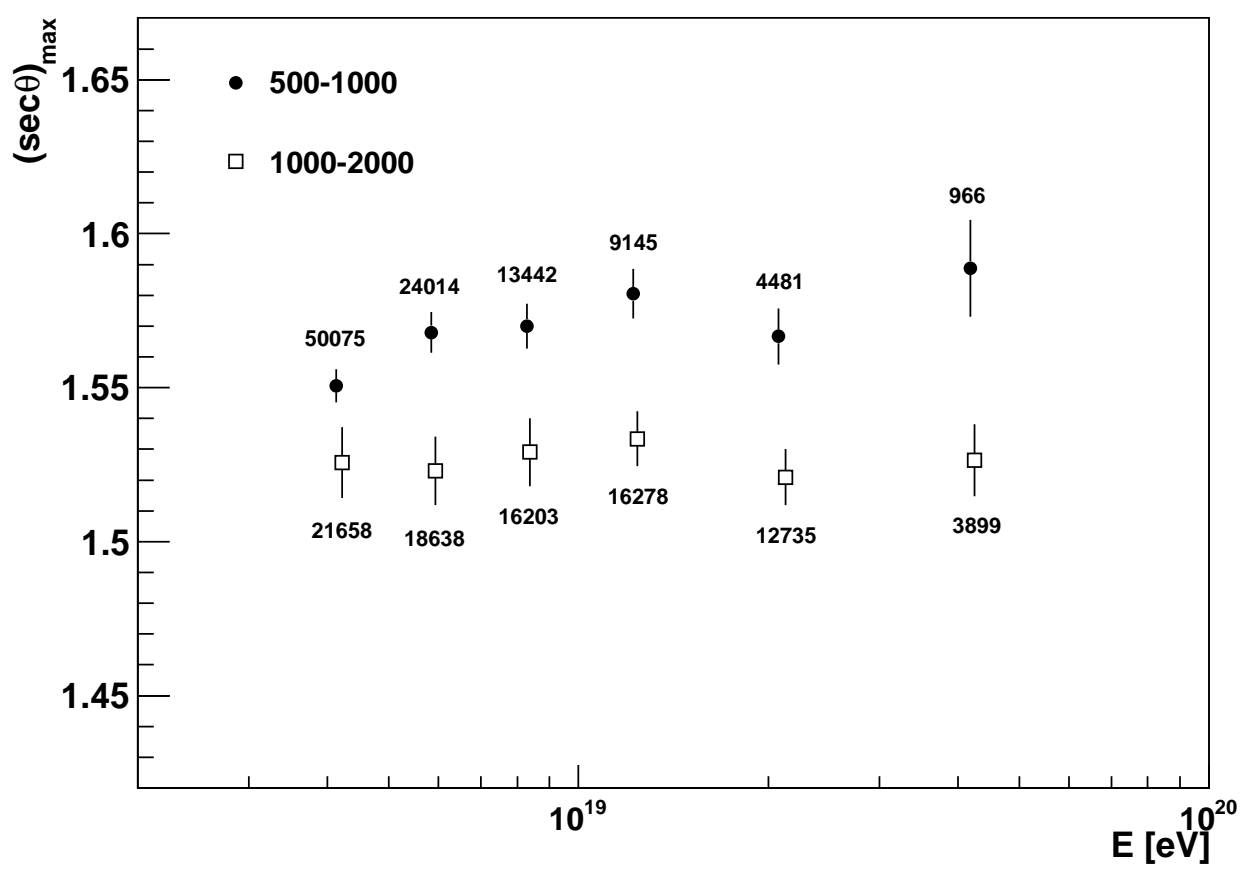

Figure 4: Energy dependence of $(\sec \theta)_{\max }$ for both intervals of core distance 500 - $1000 \mathrm{~m}$ and 1000 - 2000 $\mathrm{m}$. Number of stations available for the analysis are indicated. 

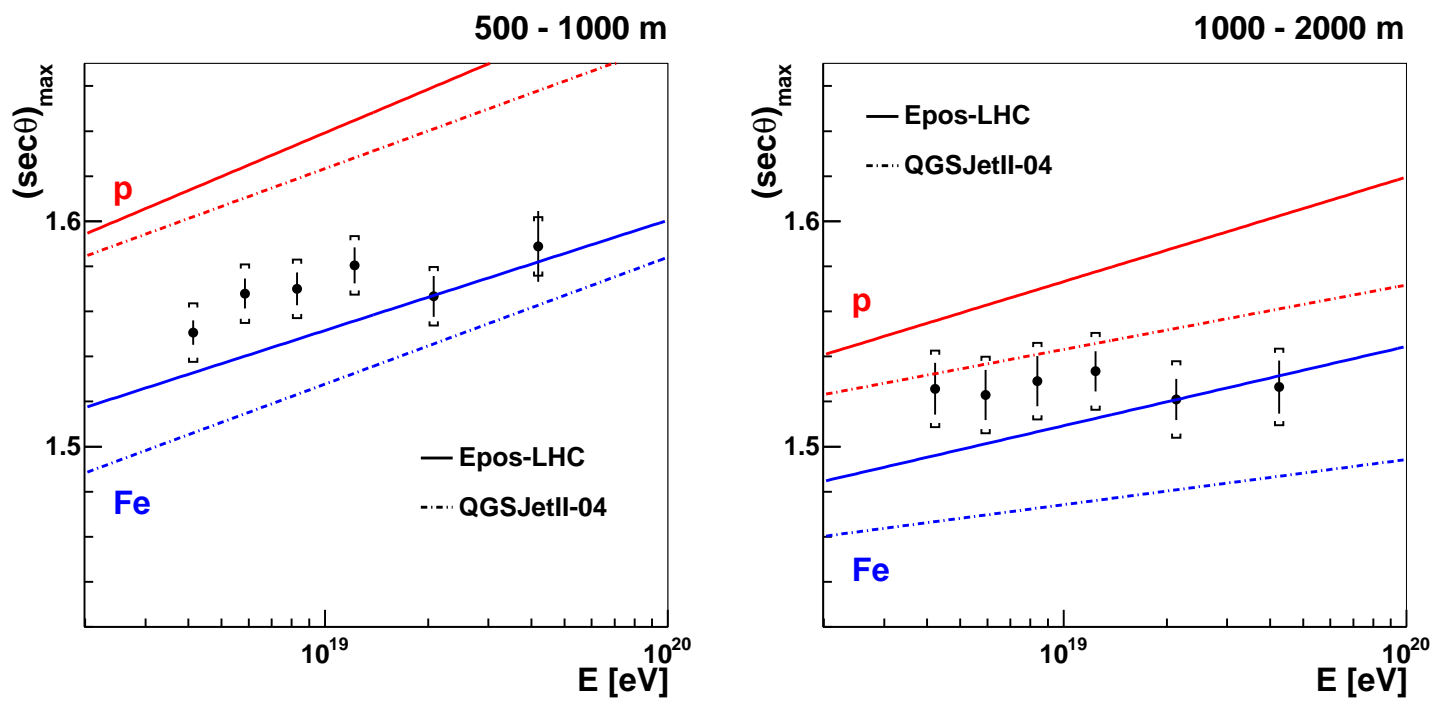

Figure 5: Comparison between $(\sec \theta)_{\max }$ for data (black points) and MC predictions using Epos-LHC (solid line) and QGSJetII-04 (dashed line) hadronic models for proton (red) and iron (blue) primaries in both $r$-intervals. Brackets represent the systematic uncertainty.

as well as the use of different parameterizations in the dependency of the risetime with $r$ and different algorithms to integrate the signal. Additionally, we have developed different cross-checks on the stability of the results, considering potential bias due to the selection cuts in the signal intensity and in the zenith interval, studying the dependence of the lateral width of the shower (in particular of the electromagnetic component) on pressure and temperature, and checking the possible effect of ageing of the SD detectors on the results. Considering all these sources we obtain a systematic uncertainty of \pm 0.013 and \pm 0.017 in units of $(\sec \theta)_{\max }$ for the $500-1000 \mathrm{~m}$ and 1000 - $2000 \mathrm{~m}$ core distance ranges respectively.

\section{Discussion}

The mass analysis has to rely on comparison with theoretical predictions using updated models of hadronic interactions extrapolated to these energies. To this end, a sample of Monte Carlo (MC) events generated with the CORSIKA [ [ 8$]$ code have been produced using the Epos-LHC [ $[$ ] and QGSJetII-04 [ए]] hadronic models for proton and iron primaries. A total of 77000 events (38500 of each primary) have been produced for each interaction model. The $\log (E / \mathrm{eV})$ values ranged from 18.00 to 20.25 in bins of 0.25 with eleven discrete $\theta$ values between $18^{\circ}$ and $63^{\circ}$.

In Fig. $\square$ the $(\sec \theta)_{\max }$ measurements as a function of energy are shown and compared with predictions made using the Epos-LHC and QGSJetII-04 models for proton and iron primaries. The dependence on energy is small and it is difficult to draw strong conclusions as rather contrasting predictions follow from the two models. However in both cases there is an indication that the mean mass increases slowly with energy in line with other Auger studies [ [ [, []].

A further illustration of the dependence of the conclusions on the choice of model is shown in Fig. 6 where the variations of $\langle\ln A\rangle$ with energy, deduced from Fig. [1, are summarised and 

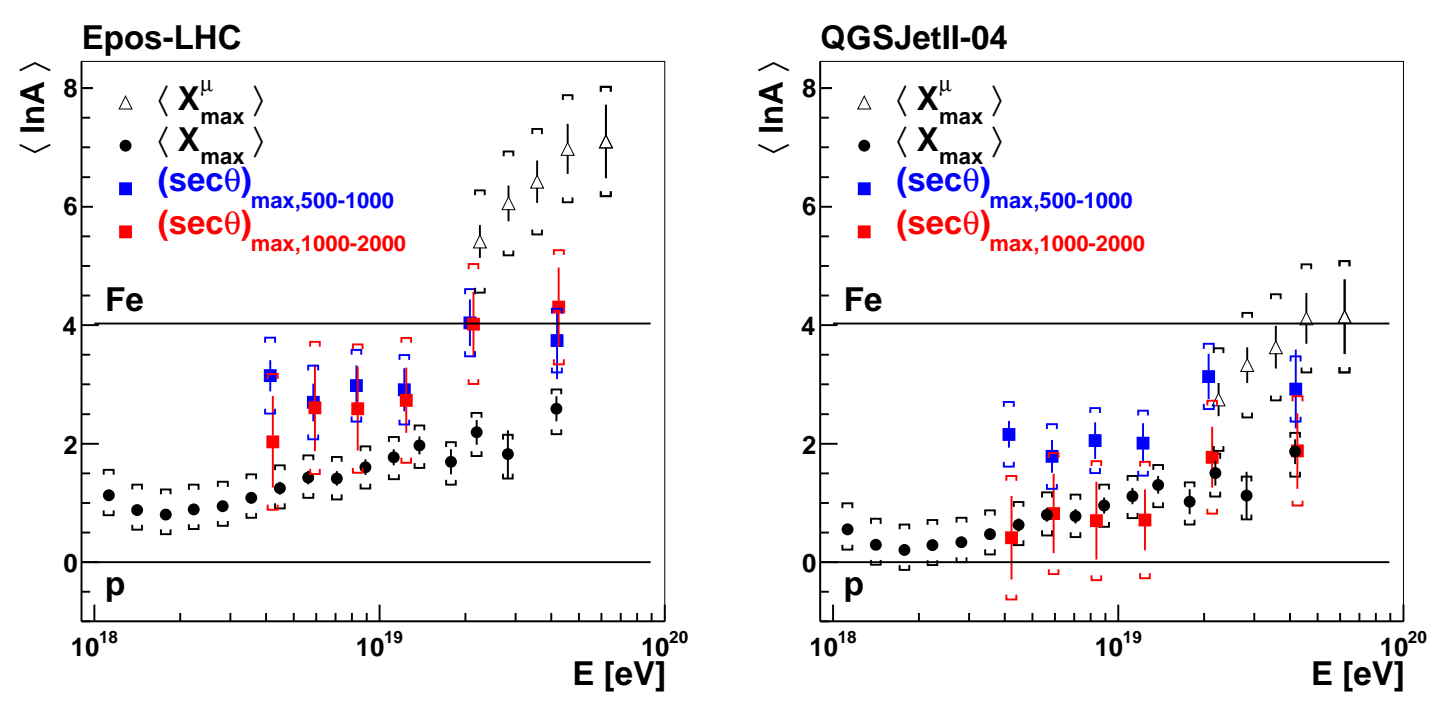

Figure 6: $\langle\ln A\rangle$ vs $E$ as predicted by Epos-LHC and QGSJetII-04. Results from the time asymmetry method in both $r$-intervals are compared with those from the elongation curve []] and the MPD method [[]].

compared with the values of $\langle\ln A\rangle$ found from the measurements of the depth of shower maximum $\left(X_{\max }\right)$ made with the fluorescence detectors [2] and using the muon production depth (MPD) technique [5].

Several inferences can be drawn from these plots. Within the uncertainties, the values of $\langle\ln A\rangle$ derived from the Epos-LHC model are consistent for the two distance ranges. However for QGSJetII-04 model this consistency is not so evident. We note that the MPD studies are of a parameter that is solely dependent on the muon content of the shower while the $X_{\max }$ observations are dominated by the electromagnetic component. As explained above, the complex nature of the zenith angle behavior of the time-asymmetry is a reflection of the contributions of both components. Thus, while the Epos-LHC is favoured over QGSJetII-04, neither model provides an accurate description of muons in air-showers. A similar conclusion has been drawn from the study of inclined showers made at the Pierre Auger Observatory [ $[\mathbb{U}]$ from which it was deduced that showers contain more muons than predicted by Epos-LHC model. Indeed no model provides a completely satisfactory description of the wide range of data using the Auger Observatory.

\section{Conclusions}

The azimuthal dependence of the $t_{1 / 2}$ values obtained from the FADC traces registered by the SD detector of the Pierre Auger Observatory has been used to obtain a mass sensitive parameter, $(\sec \theta)_{\max }$. Recent hadronic interaction models have been used to compute the $\langle\ln A\rangle$ predictions of the method described here and compare the results with those obtained from $X_{\max }$ and the MPD method. The results show a hint of a slow increase of the mean mass with energy in agreement with other studies. However, model-dependent discrepancies between data and Monte Carlo results on both core distance intervals have been found, and therefore, further deficiencies in our understand- 
ing of shower modelling have to be resolved before the mass composition can be inferred from $(\sec \theta)_{\max }$.

\section{References}

[1] The Pierre Auger Collaboration, A. Aab et al., The Pierre Auger Cosmic Ray Observatory, accepted for publication in Nucl. Instrum. Meth. A (2015) [1502.01323]].

[2] The Pierre Auger Collaboration, A. Aab et al., Depth of maximum of air-shower profiles at the Pierre Auger Observatory. I. Measurements at energies above $10^{17.8} \mathrm{eV}$, Phys. Rev. D90 (2014) 122005, [1409.4809].

[3] The Pierre Auger Collaboration, A. Porcelli, Measurements of the first two moments of the depth of shower maximum over nearly three decades of energy, combining data from the standard Pierre Auger fluorescence detector and the High Elevation Fluorescence Telescopes, these proceedings.

[4] M. Dova et al., Time asymmetries in extensive air showers: a novel method to identify UHECR species, Astropart. Phys. 31 (2009) 312-319,

[5] The Pierre Auger Collaboration, A. Aab et al., Muons in air showers at the Pierre Auger Observatory: Measurement of atmospheric production depth, Phys. Rev. D90 (2014) 012012 , Erratum: Phys. Rev. D92 (2015) 019903,

[6] The Pierre Auger Collaboration, L. Collica, Measurement of the muon content in air showers at the Pierre Auger Observatory, these proceedings.

[7] The Pierre Auger Collaboration, J. Abraham et al., Trigger and aperture of the surface detector array of the Pierre Auger Observatory, Nucl. Instrum. Meth. A613 (2010) 29-39, [[1]1.6764]].

[8] D. Heck et al., CORSIKA: A Monte Carlo code to simulate extensive air showers, FZKA-6019 (1998).

[9] T. Pierog et al., EPOS LHC : test of collective hadronization with LHC data,

[10] S. Ostapchenko, Monte Carlo treatment of hadronic interactions in enhanced Pomeron scheme: I. QGSJET-II model, Phys. Rev. D83 (2011) 014018,

[11] Pierre Auger Collaboration, A. Aab et al., Muons in air showers at the Pierre Auger Observatory: Mean number in highly inclined events, Phys. Rev. D91 (2015) 032003, [1408.1421]. 